\title{
POSITIVITY AND LINEARIZATION OF A CLASS OF NONLINEAR CONTINUOUS-TIME SYSTEMS BY STATE FEEDBACKS
}

\author{
TADEUSZ KACZOREK ${ }^{a}$ \\ ${ }^{a}$ Faculty of Electrical Engineering \\ Białystok University of Technology, ul. Wiejska 45D, 15-351 Białystok, Poland \\ e-mail: kaczorek@isep.pw.edu.pl
}

\begin{abstract}
The positivity and linearization of a class of nonlinear continuous-time system by nonlinear state feedbacks are addressed. Necessary and sufficient conditions for the positivity of the class of nonlinear systems are established. A method for linearization of nonlinear systems by nonlinear state feedbacks is presented. It is shown that by a suitable choice of the state feedback it is possible to obtain an asymptotically stable and controllable linear system, and if the closed-loop system is positive then it is unstable.
\end{abstract}

Keywords: positive, nonlinear, system, linearization, state feedback.

\section{Introduction}

In positive systems inputs, state variables and outputs take only nonnegative values. Examples of positive systems are industrial processes involving chemical reactors, heat exchangers and distillation columns, storage systems, compartmental systems, water and atmospheric pollution models. A variety of models having positive linear behavior can be found in engineering, management science, economics, social sciences, biology and medicine, etc. Positive linear systems are defined on cones and not on linear spaces. Therefore, the theory of positive systems is more complicated and less advanced.

An overview of the state of the art in positive systems theory is given by Farina and Rinaldi (2000) as well as Kaczorek (2002), who also addressed positive linear systems consisting of $n$ subsystems with different fractional orders (Kaczorek, 2011; 2012) along with minimum energy control of positive discrete-time and continuous-time linear systems (Kaczorek, 2014a; 2013; $2014 b ; 2014 c)$. The theory of the geometrical approach to the analysis of nonlinear systems based on the Lie algebra was given by Brockett (1976) and Isidori (1989). The problem of linearization of nonlinear systems by nonlinear state feedbacks was investigated by Aguilar et al. (1995), Charlet et al. (1991), Daizhan et al. (1985), Fang and Kelkar (2003), Jakubczyk (2001), Jakubczyk and Respondek (1980), Isidori (1989), Malesza (2008),
Marino and Tomei (1995), Melhem et al. (2006), Taylor and Antoniotti (1993), as well as Wei-Bing and Dang-Nan (1992).

In this paper the positivity and linearization of a class of nonlinear continuous-time systems by nonlinear state feedbacks will be addressed. The paper is organized as follows. In Section 2, necessary and sufficient conditions for the positivity of a class of nonlinear systems are established. Linearization of the nonlinear system by a nonlinear state feedback is addressed in Section 3. An example illustrating the discussion is given in Section 4. Concluding remarks are presented in Section 5.

The following notation will be used: $\mathbb{R}$, the set of real numbers; $\mathbb{R}^{n \times m}$, the set of $n \times m$ real matrices and $\mathbb{R}^{n}=$ $\mathbb{R}^{n \times 1} ; \mathbb{R}_{+}^{n \times m}$, the set of $n \times m$ matrices with nonnegative entries and $\mathbb{R}_{+}^{n}=\mathbb{R}_{+}^{n \times 1} ; M_{n}$, the set of $n \times n$ Metzler matrices (with nonnegative off-diagonal entries); $I_{n}$, the $n \times n$ identity matrix.

\section{Positivity of nonlinear systems}

Consider the nonlinear system

$$
\dot{x}=A x+f(x)+B u,
$$


where

$$
\begin{gathered}
x=\left[\begin{array}{c}
x_{1} \\
\vdots \\
x_{n}
\end{array}\right], \quad A=\left[\begin{array}{ccccc}
0 & 1 & 0 & \ldots & 0 \\
0 & 0 & 1 & \ldots & 0 \\
\vdots & \vdots & \vdots & \ddots & \vdots \\
0 & 0 & 0 & \ldots & 1 \\
0 & 0 & 0 & \ldots & 0
\end{array}\right], \\
f(x)=\left[\begin{array}{c}
f_{1}\left(x_{1}\right) \\
f_{2}\left(x_{1}, x_{2}\right) \\
\vdots \\
f_{n}\left(x_{1}, \ldots, x_{n}\right)
\end{array}\right], \quad B=\left[\begin{array}{c}
0 \\
\vdots \\
0 \\
1
\end{array}\right],
\end{gathered}
$$

$x=x(t) \in \mathbb{R}^{n}, u=u(t) \in \mathbb{R}$ are the state vector and the input vector, respectively.

It is assumed that the functions $f_{k}\left(x_{1}, \ldots, x_{k}\right), k=$ $1,2, \ldots, n$, are continuously differentiable for all their arguments.

Definition 1. The nonlinear system (11) is called (internally) positive if $x(t) \in \mathbb{R}_{+}^{n}$ for all $x(0) \in \mathbb{R}_{+}^{n}, t \geq 0$ and every $u(t) \in \mathbb{R}_{+}, t \geq 0$.

Theorem 1. The nonlinear system (1) is positive if and only if

$$
\left.\begin{array}{l}
f_{k}(\bar{x}) \in \mathbb{R}_{+} \text {for } \\
\quad \bar{x}=\left[x_{1}, \ldots, x_{j-1}, 0, x_{j+1}, \ldots, x_{k}(t)\right]^{T} \in \mathbb{R}_{+}, \\
j=1,2, \ldots, k \text { and } u(t) \in \mathbb{R}_{+}, t \geq 0 .
\end{array}\right\}
$$

Proof. For given $f(x)$, the solution of (1) has the form

$$
x(t)=e^{A t} x(0)+\int_{0}^{t} e^{A(t-\tau)}[f(x(\tau))+B u(\tau)] \mathrm{d} \tau .
$$

The linear system obtained from (1) for $f_{k}\left(x_{1}, \ldots, x_{k}\right)=$ $0, k=1,2, \ldots, n$, is positive since the matrix $A$ is a Meltzer matrix, $B \in \mathbb{R}_{+}^{n}$.

Using the well-known Picard method, the $k$-approximation of the solution of (1) can be found from the formula

$$
\begin{aligned}
x_{k+1}(t)= & e^{A t} x(0) \\
& +\int_{0}^{t} e^{A(t-\tau)}\left[f\left(x_{k}(\tau)\right)+B u(\tau)\right] \mathrm{d} \tau
\end{aligned}
$$

for $k=1,2, \ldots$.

The Lipschitz conditions for (1) are satisfied since, by assumption, the functions $f_{k}\left(x_{1}, \ldots, x_{k}\right), k=$ $1,2, \ldots, n$, are continuously differentiable. Using the Picard method, it is easy to show that Eqn. (1) has nonnegative solution $x(t) \in \mathbb{R}_{+}^{n}, t \geq 0$ if and only if the conditions (3) are satisfied.

The proof can be also accomplished using the method presented by Malesza and Respondek (2007).

\section{Linearization by state feedbacks}

For the nonlinear system (1), we introduce the following new state variables (the components of the new state vector $\left.z=\left[\begin{array}{lll}z_{1} & \ldots & z_{n}\end{array}\right]^{T}\right)$ :

$$
\begin{aligned}
z_{1}= & x_{1}, \\
z_{2}= & x_{2}+f_{1}\left(x_{1}\right), \\
z_{3}= & x_{3}+f_{2}\left(x_{1}, x_{2}\right)+\frac{\partial f_{1}}{\partial x_{1}}\left[x_{2}+f_{1}\left(x_{1}\right)\right] \\
= & x_{3}+\bar{f}_{2}\left(x_{1}, x_{2}\right), \\
z_{4}= & x_{4}+f_{3}\left(x_{1}, x_{2}, x_{3}\right)+\frac{\partial \bar{f}_{2}}{\partial x_{1}}\left[x_{2}+f_{1}\left(x_{1}\right)\right] \\
& +\frac{\partial \bar{f}_{2}}{\partial x_{2}}\left[x_{3}+f_{2}\left(x_{1}, x_{2}\right)\right] \\
= & x_{4}+\bar{f}_{3}\left(x_{1}, x_{2}, x_{3}\right), \\
\vdots & \\
z_{n}= & x_{n}+\bar{f}_{n-1}\left(x_{1}, \ldots, x_{n-1}\right) .
\end{aligned}
$$

The relations (6) can be written shortly as $z=\phi(x)$.

From [6, we have

$$
\begin{aligned}
x_{1} & =z_{1}, \\
x_{2} & =z_{2}-f_{1}\left(z_{1}\right), \\
x_{3} & =z_{3}-\bar{f}_{2}\left(z_{1}, z_{2}\right), \\
& \vdots \\
x_{n} & =z_{n}-\bar{f}_{n-1}\left(z_{1}, \ldots, z_{n-1}\right) .
\end{aligned}
$$

This can be briefly expressed as $x=\phi^{-1}(z)$.

The nonlinear system (1) in the new state variables (6) has the form

$$
\begin{aligned}
\dot{z}_{1}= & \dot{x}_{1}=x_{2}+f_{1}\left(x_{1}\right)=z_{2}, \\
\dot{z}_{2}= & \dot{x}_{2}+\frac{\partial f_{1}}{\partial x_{1}} \dot{x}_{1}=x_{3}+f_{2}\left(x_{1}, x_{2}\right) \\
& +\frac{\partial f_{1}}{\partial x_{1}}\left[x_{2}+f_{1}\left(x_{1}\right)\right]=z_{3}, \\
\vdots & \\
\dot{z}_{n-1}= & x_{n}+f_{n-1}\left(x_{1}, \ldots, x_{n-1}\right) \\
& +\frac{\partial \bar{f}_{n-2}}{\partial x_{1}}\left[x_{2}+f_{1}\left(x_{1}\right)\right]+\cdots+\frac{\partial \bar{f}_{n-2}}{\partial x_{n-2}} \\
& \times\left[x_{n-1} f_{n-2}\left(x_{1}, \ldots, x_{n-2}\right)\right]=z_{n}, \\
\dot{z}_{n}= & f_{n}\left(x_{1}, \ldots, x_{n}\right)+u+\frac{\partial \bar{f}_{n-1}}{\partial x_{1}}\left[x_{2}+f_{1}\left(x_{1}\right)\right] \\
& +\cdots+\frac{\partial \bar{f}_{n-1}}{\partial x_{n-1}} \\
& \times\left.\left[x_{n}+f_{n-1}\left(x_{1}, \ldots, x_{n-1}\right)\right]\right|_{x=\phi^{-1}(z)} \\
= & -a_{0} z_{1}-a_{1} z_{2}-\ldots-a_{n-1} z_{n}+v,
\end{aligned}
$$


where

$$
\begin{aligned}
v= & u+g(x) \\
g(x)= & \left.\sum_{i=0}^{n-1} a_{i} z_{i+1}\right|_{z=\phi(x)}+f_{n}\left(x_{1}, \ldots, x_{n}\right) \\
& +\frac{\partial \bar{f}_{n-1}}{\partial x_{1}}\left[x_{2}+f_{1}\left(x_{1}\right)\right] \\
& +\cdots+\frac{\partial \bar{f}_{n-1}}{\partial x_{n-1}}\left[x_{n}+f_{n-1}\left(x_{1}, \ldots, x_{n-1}\right)\right] .
\end{aligned}
$$

Equations (8) can be written in the form

$$
\dot{z}=A z+B v, \quad z(0)=\phi[x(0)] \in \mathbb{R}^{n},
$$

where

$$
\begin{aligned}
& \bar{A}=\left[\begin{array}{ccccc}
0 & 1 & 0 & \ldots & 0 \\
0 & 0 & 1 & \ldots & 0 \\
\vdots & \vdots & \vdots & \ddots & \vdots \\
0 & 0 & 0 & \ldots & 1 \\
-a_{0} & -a_{1} & -a_{2} & \ldots & -a_{n-1}
\end{array}\right], \\
& B=\left[\begin{array}{c}
0 \\
\vdots \\
0 \\
1
\end{array}\right] .
\end{aligned}
$$

Note that applying to the nonlinear system (8) the nonlinear state feedback

$$
u=v-g(x),
$$

we obtain the linear closed-loop system described by Eqn. (10).

The coefficients $a_{k}, k=0,1, \ldots, n-1$, can be chosen so that the linear system (10) is asymptotically stable.

For all values of the coefficients $a_{k}, k=$ $0,1, \ldots, n-2$, the pair 111 is controllable since (Jakubczyk and Respondek, 1980; Isidori, 1989)

$$
\operatorname{rank}\left[\begin{array}{cccc}
B & \bar{A} B & \ldots & \bar{A}^{n-1} B
\end{array}\right]=n .
$$

Note that the linear system (10) with (11) is positive if and only if $a_{k}=0, k=0,1, \ldots, n-1$. In this case, the linear system is unstable.

Therefore, the following results have been proven.

Theorem 2. The nonlinear system (1) can be linearized by the nonlinear state feedback (12) and for a suitable choice of the coefficients $a_{k}, k=0,1, \ldots, n-1$, the linear closed-loop system (10) is asymptotically stable and controllable.

Theorem 3. The nonlinear system (1) can be linearized by the nonlinear state feedback (12), so that the closedloop system (10) for $a_{k}=0, k=0,1, \ldots, n-1$, is positive but unstable.

\section{Example}

Consider the nonlinear system described by the equations

$$
\dot{x}=\left[\begin{array}{c}
\dot{x}_{1} \\
\dot{x}_{2} \\
\dot{x}_{3}
\end{array}\right]=\left[\begin{array}{c}
x_{2}+x_{1}^{2} \\
x_{3}+x_{1} x_{2} \\
x_{2} x_{3}+u
\end{array}\right], \quad x(0) \in \mathbb{R}_{+}^{3} .
$$

The system (14) is positive since Eqn. (14) satisfies the conditions (3) and $u=u(t) \in \mathbb{R}_{+}, t \geq 0$. In this case, the new state variables $z_{k}, k=1,2,3$, are defined as follows:

$$
z=\left[\begin{array}{c}
z_{1} \\
z_{2} \\
z_{3}
\end{array}\right]=\left[\begin{array}{c}
x_{1} \\
x_{2}+x_{1}^{2} \\
x_{3}+3 x_{1} x_{2}+2 x_{1}^{3}
\end{array}\right]=\phi(x)
$$

and

$$
x=\left[\begin{array}{l}
x_{1} \\
x_{2} \\
x_{3}
\end{array}\right]=\left[\begin{array}{c}
z_{1} \\
z_{2}-z_{1}^{2} \\
z_{3}-3 z_{1} z_{2}+z_{1}^{3}
\end{array}\right] .
$$

The nonlinear system (14) in the new state variables is described by Eqn. (17).

To linearize the nonlinear system (17), we apply the nonlinear state feedback (12) of the form

$$
\begin{aligned}
u= & v-g(z) \\
= & v-a_{0} z_{1}-a_{1} z_{2}-a_{2} z_{3}+z_{1}^{5}+2 z_{1}^{4}-4 z_{1}^{3} z_{2} \\
& +3 z_{1}^{2} z_{2}+z_{1}^{2} z_{3}-3 z_{2}^{2}+3 z_{1} z_{2}^{2} \\
& -3 z_{1} z_{3}-z_{2} z_{3},
\end{aligned}
$$

and we obtain the linear system (10) with

$$
A=\left[\begin{array}{ccc}
0 & 1 & 0 \\
0 & 0 & 1 \\
-a_{0} & -a_{1} & -a_{2}
\end{array}\right], \quad B=\left[\begin{array}{l}
0 \\
0 \\
1
\end{array}\right]
$$

The linear system is controllable for all values of the coefficients $a_{k}, k=0,1,2$, and it is asymptotically stable if and only if $a_{k}>0, k=0,1,2$, and $a_{1} a_{2}>a_{0}$.

The linear system (10) with (19) is positive if and only if $a_{k}=0, k=0,1$ since in this case

$$
A=\left[\begin{array}{lll}
0 & 1 & 0 \\
0 & 0 & 1 \\
0 & 0 & 0
\end{array}\right] \in M_{3}
$$

In this case the linear system is unstable.

\section{Concluding remarks}

The positivity and linearization of a class of nonlinear systems by nonlinear state feedbacks were addressed. Necessary and sufficient conditions for the positivity of the class of nonlinear systems (Theorem 1) were established. It was shown that the nonlinear systems can be linearized by nonlinear feedbacks, so that the 


$$
\dot{z}=\left[\begin{array}{c}
\dot{z}_{1} \\
\dot{z}_{2} \\
\dot{z}_{3}
\end{array}\right]=\left[\begin{array}{c}
z_{2} \\
z_{3} \\
-z_{1}^{5}-2 z_{1}^{4}+4 z_{1}^{3} z_{2}-3 z_{1}^{2} z_{2}-z_{1}^{2} z_{3}+3 z_{2}^{2}-3 z_{1} z_{2}^{2}+3 z_{1} z_{3}+z_{2} z_{3}+u
\end{array}\right]
$$

linear close-loop system is asymptotically stable and controllable (Theorem 2) and positive but unstable (Theorem 3). The discussion was illustrated by an example. An open problem is the extension of these deliberations to fractional nonlinear systems.

\section{Acknowledgment}

This work was supported by the National Science Centre in Poland under the work G/WE/1/2015.

\section{References}

Aguilar, J.L.M., Garcia, R.A. and D'Attellis, C.E. (1995). Exact linearization of nonlinear systems: Trajectory tracking with bounded control and state constrains, 38th Midwest Symposium on Circuits and Systems, Rio de Janeiro, Brazil, pp. 620-622.

Brockett, R.W. (1976). Nonlinear systems and differential geometry, Proceedings of the IEEE 64(1): 61-71.

Charlet, B., Levine, J. and Marino, R. (1991). Sufficient conditions for dynamic state feedback linearization, SIAM Journal on Control and Optimization 29(1): 38-57.

Daizhan, C., Tzyh-Jong, T. and Isidori, A. (1985). Global external linearization of nonlinear systems via feedback, IEEE Transactions on Automatic Control 30(8): 808-811.

Fang, B. and Kelkar, A.G. (2003). Exact linearization of nonlinear systems by time scale transformation, $I E$ EE American Control Conference, Denver, CO, USA, pp. 3555-3560.

Farina, L. and Rinaldi, S. (2000). Positive Linear Systems: Theory and Applications, J. Wiley, New York, NY.

Isidori, A. (1989). Nonlinear Control Systems, Springer-Verlag, Berlin.

Jakubczyk, B. (2001). Introduction to geometric nonlinear control: Controllability and Lie bracket, Summer School on Mathematical Control Theory, Triest, Italy.

Jakubczyk, B. and Respondek, W. (1980). On linearization of control systems, Bulletin of the Polish Academy Sciences: Technical Sciences 28: 517-521.

Kaczorek, T. (2002). Positive $1 D$ and $2 D$ Systems, Springer Verlag, London.

Kaczorek, T. (2011). Positive linear systems consisting of $n$ subsystems with different fractional orders, IEEE Transactions on Circuit and Systems 58(6): 1203-1210.

Kaczorek, T. (2012). Selected Problems of Fractional System Theory, Springer Verlag, Berlin.
Kaczorek, T. (2013). Minimum energy control of fractional positive discrete-time linear systems, Bulletin of the Polish Academy of Sciences: Technical Sciences 61(4): 803-807.

Kaczorek, T. (2014a). Minimum energy control of descriptor positive discrete-time systems, COMPEL 33(3): 1-14.

Kaczorek, T. (2014b). Necessary and sufficient conditions for minimum energy control of positive discrete-time linear systems with bounded inputs, Bulletin of the Polish Academy of Sciences: Technical Sciences 62(1): 85-89.

Kaczorek, T. (2014c). Minimum energy control of fractional positive continuous-time linear systems with bounded inputs, International Journal of Applied Mathematics and Computer Science 24(2): 335-340, DOI: 10.2478/amcs-2014-0025.

Malesza, W. (2008). Geometry and Equivalence of Linear and Nonlinear Control Systems Invariant on Corner Regions, Ph.D. thesis, Warsaw University of Technology, Warsaw.

Malesza, W. and Respondek, W. (2007). State-linearization of positive nonlinear systems: Applications to Lotka-Volterra controlled dynamics, in F. Lamnabhi-Lagarrigu et al. (Eds.), Taming Heterogeneity and Complexity of Embedded Control, John Wiley, Hoboken, NJ, pp. 451-473.

Marino, R. and Tomei, P. (1995). Nonlinear Control DesignGeometric, Adaptive, Robust, Prentice Hall, London.

Melhem, K., Saad, M. and Abou, S.C. (2006). Linearization by redundancy and stabilization of nonlinear dynamical systems: A state transformation approach, IEEE International Symposium on Industrial Electronics, Montreal, Canada, pp. 61-68.

Taylor, J.H. and Antoniotti, A.J. (1993). Linearization algorithms for computer-aided control engineering, Control Systems Magazine 13(2): 58-64.

Wei-Bing, G. and Dang-Nan, W. (1992). On the method of global linearization and motion control of nonlinear mechanical systems, International Conference on Industrial Electronics, Control, Instrumentation and Automation, San Diego, CA, USA, pp. 1476-1481.

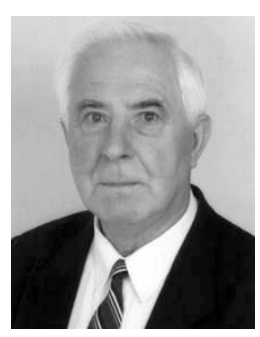

Tadeusz Kaczorek received the M.Sc., Ph.D and D.Sc. degrees in electrical engineering from the Warsaw University of Technology in 1956, 1962 and 1964, respectively. In the years 1968-69 he was the dean of the Electrical Engineering Faculty, and in the period of 1970-73 he was a deputy rector of the Warsaw University of Technology. In 1971 he became a professor and in 1974 a full professor at the same university. Since 2003 he has been a professor at the Białystok University of Technology. In 1986 he was elected a corresponding member and in 1996 a full member of the Polish Academy of Sciences. In the years 1988-1991 he was the director of the Research Center of 
the Polish Academy of Sciences in Rome. In 2004 he was elected an honorary member of the Hungarian Academy of Sciences. He was granted honorary doctorates by 13 universities. His research interests cover systems theory, especially singular multidimensional systems, positive multidimensional systems, singular positive 1D and 2D systems, as well as positive fractional $1 \mathrm{D}$ and $2 \mathrm{D}$ systems. He initiated research in the field of singular 2D, positive 2D and positive fractional linear systems. He published 28 books ( 8 in English) and over 1100 scientific papers. He also supervised $69 \mathrm{Ph} . \mathrm{D}$. theses. He is the editor-in-chief of the Bulletin of the Polish Academy of Sciences: Technical Sciences and a member of editorial boards of ten international journals.

Received: 22 August 2014

Revised: 24 January 2015

Re-revised: 10 March 2015 